\title{
Resonances in the dynamics of $\phi^{4}$ kinks perturbed by ac forces
}

\author{
Niurka R. Quintero* and Angel Sánchez ${ }^{\dagger}$ \\ Grupo Interdisciplinar de Sistemas Complicados (GISC), Departamento de Matemáticas, Universidad Carlos III de Madrid, \\ Edificio Sabatini, Avenida de la Universidad 30, E-28911 Leganés, Madrid, Spain \\ Franz G. Mertens \\ Physikalisches Institut, Universität Bayreuth, D-95440 Bayreuth, Germany
}

(Received 13 March 2000)

\begin{abstract}
We study the dynamics of $\phi^{4}$ kinks perturbed by an ac force, both with and without damping. We address this issue by using a collective coordinate theory, which allows us to reduce the problem to the dynamics of the kink center and width. We carry out a careful analysis of the corresponding ordinary differential equations, of Mathieu type in the undamped case, finding and characterizing the resonant frequencies and the regions of existence of resonant solutions. We verify the accuracy of our predictions by numerical simulation of the full partial differential equation, showing that the collective coordinate prediction is very accurate. Numerical simulations for the damped case establish that the strongest resonance is the one at half the frequency of the internal mode of the kink. In conclusion, we discuss the possible relevance of our results for other systems, especially the sine-Gordon equation. We also obtain additional results regarding the equivalence between different collective coordinate methods applied to this problem.

PACS number(s): 05.45.Yv, 02.30.Jr, 03.50.-z, 63.20.Pw
\end{abstract}

\section{INTRODUCTION}

A century and a half after their discovery, solitons and solitary waves have proven themselves ubiquitous in nature, arising in many physical applications and leading to very important advances in applied mathematics [1,2]. Generally speaking, it is often the case that the properties of solitary waves are known for certain equations, perhaps integrable, that relate to an oversimplified description of different physical systems; subsequently, one is interested to learn how these properties are modified if terms initially neglected are included as perturbations of the original equation. In most cases, this is a very complicated problem, and shedding light on it usually requires the use of approximate analytical approaches. One of the most succesful and widely applicable of these approaches is the collective coordinate technique or, rather, the family of collective coordinate techniques $[3,4]$. The main merit of these procedures is the drastic reduction of the number of degrees of freedom involved in the problem, from the multiplicity of them in the original partial differential equation to the dynamics of a few degrees of freedom, governed by ordinary differential equations. Quite commonly, the reduction is done to a single degree of freedom, which, in general, can be identified with the center of the wave $X(t)$ or its velocity $V(t)$, as first proposed in the mid1970 s, $[5,6]$. This amounts to mapping the motion of solitons or solitary waves to the motion of a pointlike (perhaps relativistic) particle with an effective mass [7]. Surprisingly, this dramatic simplification leads to excellent results for many systems $[3,4]$. However, there are perturbations which, when acting on solitary waves, change not only the position of the

\footnotetext{
*Email address: kinter@math.uc3m.es

${ }^{\dagger}$ Email address: anxo@math.uc3m.es

†Email address: franz mertens@theo.phy.uni-bayreuth.de
}

center of mass of the kink but also its width, as suggested in Ref. [8]. In addition, some radiation can appear in the waves, a phenomenon that can render the whole collective coordinate idea useless as more and more degrees of freedom are excited $[9,10]$.

As mentioned above, in 1983 Rice [8] developed a new perturbative method, which he applied to two well-known nonlinear Klein-Gordon problems, the $\phi^{4}$ and the sineGordon equations $[1,2]$, in order to account for variations of the width of their kink solutions under perturbations. In Rice's approach, the collective coordinates are the kink center $X(t)$ and its width $l(t)$. His results pointed out that when, in those systems, the kink is subject to "some perturbations" (sic) the simple translational motion of the kink center can be coupled to an oscillatory motion of the width of the wave, whose frequency he obtained by means of a variational approach. Interestingly, for the $\phi^{4}$ equation this so-called Rice frequency $\Omega_{R}$ practically coincides with the frequency $\Omega_{i}$ of the kink internal mode (one of the modes of linear excitations around the kink, corresponding to a nonzero eigenvalue in the discrete spectrum; see, e.g., Ref. [11]). Conversely, for the sine-Gordon ( $\mathrm{sG}$ ) case, $\Omega_{R}$ turns out to be within the phonon spectrum and, furthermore, the $\mathrm{sG}$ kink does not have an internal mode (its only eigenvalue in the discrete spectrum is zero, corresponding to a Goldstone mode [5]). For this reason, the deformation of the kink width due to the internal mode was studied extensively in the $\phi^{4}$ equation and, among other interesting results, we now know that the internal mode is able to store and transfer the energy in $\phi^{4}$ kink-antikink collisions [12], in the interaction of $\phi^{4}$ kinks with impurities [13], or in the case when the $\phi^{4}$ kink is subject to a periodic spatially modulated potential [14]. This exchange of energy between the internal and translational modes, or among the internal mode and the modes of the impurities, explains the resonances that take place in the above perturbed $\phi^{4}$ systems. 
In view of these results, a question that naturally arises is the possible existence of resonances of the $\phi^{4}$ kink when perturbed by external ac forces: a problem that has not been considered so far, and that relates to a number of physical contexts. Very recently, we showed [15] that a strong resonance arises when the $\phi^{4}$ system is subject to an external ac force of the form $f(t)=\epsilon \sin \left(\delta t+\delta_{0}\right)$, with $\delta$ close to $\Omega_{i} / 2$, while in the case that $\delta=\Omega_{i}$ this resonance is weak and even disappears for an appropriate choice of the initial kink velocity and the parameters of the driving force. However, the work reported in Ref. [15] contained mostly numerical results, and only an intuitive explanation of this striking phenomenon in terms of the collective coordinate equations; therefore, our aim in this paper is to provide a full analytical treatment of those equations (for the undamped case) in order to better understand the anomalous resonance phenomenon. In addition, in doing so we will be already carrying out the same analysis for the sG equation, which may be of interest in order to clarify the question of the existence of an internal quasimode for this equation [16]. We deal with these issues throughout the paper according to the following scheme: In Sec. II we use the so called generalized travelling wave ansatz (GTWA) [17] to obtain the equations governing the dynamics of the kink center and width (which is associated to the excitation of the internal mode). In Sec. III we thoroughly analyze those equations in the absence of damping, identifying all the possible resonances and their locations as a function of the equation parameters. We then focus on the most interesting resonances, namely, those at $\Omega_{i} / 2$ and $\Omega_{i}$. For the damped case we have not been able to solve the equation for $l(t)$ analytically, but in Sec. IV we present numerical simulations of the full partial differential equation, confirming that the resonance at $\Omega_{i} / 2$ remains for weak damping, while the resonance at $\delta \approx \Omega_{i}$ disappears for any damping value. Additionally, in that section we compare all our analytical results to the numerical simulations, finding excellent agreement. Finally, in Sec. V we summarize our results and discuss their implications for other systems, focusing especially on the sG kink dynamics. In an appendix, we prove the equivalence of the GTWA to a different procedure to obtain collective coordinate equations, which uses the momentum and the energy of the system similarly to the classical approach in [6].

\section{COLLECTIVE COORDINATE APPROACH}

The dynamics of $\phi^{4}$ kinks subject to a periodic force $f(t)=\epsilon \sin \left(\delta t+\delta_{0}\right)$ is governed by the equation

$$
\phi_{t t}-\phi_{x x}-\phi+\phi^{3}=-\beta \phi_{t}+f(t),
$$

where $\beta$ is the damping coefficient, and $\epsilon, \delta$, and $\delta_{0}$ represent the amplitude, frequency, and phase of the periodic force, respectively.

In order to apply the GTWA, first proposed in Ref. [17], we rewrite Eq. (1) in the following way:

$$
\dot{\phi}=\frac{\delta H}{\delta \psi}
$$

$$
\dot{\psi}=-\frac{\delta H}{\delta \phi}+F\left(x, t, \phi, \phi_{t}, \ldots\right),
$$

where $\psi=\dot{\phi}$, the dot represents the derivative with respect to time, $F\left(x, t, \phi, \phi_{t}, \ldots\right)=-\beta \dot{\phi}+f(t)$, and $H$ is the Hamiltonian of the system with the corresponding potential $U(\phi)$ $=\left(\phi^{2}-1\right)^{2} / 4$ when $\epsilon$ and $\beta$ are zero:

$$
H=\int_{-\infty}^{+\infty} d x\left\{\frac{1}{2} \psi^{2}+\frac{1}{2} \phi_{x}^{2}+U(\phi)\right\}
$$

We now assume that the solution of Eqs. (2) and (3) has the form

$$
\phi(x, t)=\phi[x-X(t), l(t)]
$$

and, hence, from the definition of $\psi$ we have that

$$
\psi(x, t)=\psi[x-X(t), l(t), \dot{X}, i] .
$$

Here $\phi$ describes the soliton shape, whose center will be given by $X(t)$, and where we introduced a second collective variable $l(t)$ which will represent the kink width, as we see below. This ansatz takes into account that $l$ can be different from the Lorentz-contracted width due to the action of the external force. Since the internal mode is related with the kink width, we can expect that by using this approach we will be able to explain the kink motion when the internal mode is excited. However, the linearized problem around the initial kink solution tells us that perturbations of the kink cannot only shift its position or change its width, but also make it emit radiation [11]. We have not considered this effect, and therefore the ansatz is only valid when the external force is so small that practically no radiation is emitted. Since the frequencies of the discrete internal mode $\Omega_{i}$ $=\sqrt{3 / 2}$ and the continuum phonon band $\omega_{p}=\sqrt{2+k^{2} / 2}$ are separated from each other, one can expect that the two kinds of modes are excited at different values of the parameters of the external force; then, when resonances appear as a consequence of the excitation of the internal mode, one can in principle neglect the effect of radiation. In any event all our analytical results will have to be confirmed later by numerical simulations, which will show whether or not this assumption is correct.

To obtain the equations for our collective coordinates $X(t)$ and $l(t)$, we will follow Ref. [17] (see the Appendix for another possible derivation). First, we insert Eqs. (5) and (6) into Eq. (2) and (3), and then multiply the first obtained equation by $\partial \psi / \partial X$ and the second by $\partial \phi / \partial X$; subtracting both expressions and integrating we arrive at the system of equations

$$
\begin{gathered}
\int_{-\infty}^{+\infty} d x \frac{\partial \phi}{\partial X} \frac{\partial \psi}{\partial \dot{X}} \ddot{X}+\int_{-\infty}^{+\infty} d x[\phi, \psi] \dot{l}+\int_{-\infty}^{+\infty} d x \frac{\partial \phi}{\partial X} \frac{\partial \psi}{\partial \dot{l}} \\
-F^{\text {stat }}(X)=\int_{-\infty}^{+\infty} d x F\left(x, t, \phi, \phi_{t}, \ldots\right) \frac{\partial \phi}{\partial X}
\end{gathered}
$$




$$
\begin{gathered}
{[\phi, \psi]=\frac{\partial \phi}{\partial X} \frac{\partial \psi}{\partial l}-\frac{\partial \phi}{\partial l} \frac{\partial \psi}{\partial X},} \\
F^{s t a t}=-\int_{-\infty}^{+\infty} d x\left\{\frac{\delta H}{\delta \phi} \frac{\partial \phi}{\partial X}+\frac{\delta H}{\delta \psi} \frac{\partial \psi}{\partial X}\right\} \\
=-\int_{-\infty}^{+\infty} d x \frac{\partial \mathcal{H}}{\partial X}=-\frac{\partial E}{\partial X}
\end{gathered}
$$

where $E$ represents the energy of the system, $\mathcal{H}$ is the Hamiltonian density, and $F^{\text {stat }}$ is the static force due the external field or other solitons, equal to zero for the above Hamiltonian. In order to obtain the second equation of motion, we proceed as in the previous equation and begin by inserting Eqs. (5) and (6) into Eqs. (2) and (3); then we multiply the first and second equations by $\partial \psi / \partial l$ and $\partial \phi / \partial l$, respectively; finally we take the difference and integrate, thus finding

$$
\begin{gathered}
\int_{-\infty}^{+\infty} d x[\psi, \phi] \dot{X}+\int_{-\infty}^{+\infty} d x \frac{\partial \phi}{\partial l} \frac{\partial \psi}{\partial \dot{X}} \ddot{X} \\
+\int_{-\infty}^{+\infty} d x \frac{\partial \phi}{\partial l} \frac{\partial \psi}{\partial \dot{l}} \ddot{l}-K^{i n t}(X) \\
=\int_{-\infty}^{+\infty} d x F\left(x, t, \phi, \phi_{t}, \ldots\right) \frac{\partial \phi}{\partial l},
\end{gathered}
$$

where

$$
K^{i n t}(l, \dot{l}, \dot{X})=-\int_{-\infty}^{+\infty} d x \frac{\partial \mathcal{H}}{\partial l}=-\frac{\partial E}{\partial l} .
$$

By this procedure, we have obtained two coupled secondorder ordinary differential equations for $X(t)$ and $l(t)$, where up to now we have not imposed any condition on the soliton shape; however, to solve Eqs. (7)-(11) we need an explicit functional dependence of $\phi(x, t)$. Following Rice [8], we assume that

$$
\phi(x, t)=\phi_{0}[x-X(t), l(t)]=\tanh \left[\frac{x-X(t)}{l(t)}\right]
$$

where $\phi_{0}=\tanh \left[x-X_{0} / l_{0}\right]$ is the static kink solution of the $\phi^{4}$ system, centered at $X_{0}$ and of width $l_{0}=\sqrt{2}$. Substituting Eq. (12) into Eqs. (7)-(11) and integrating over $x$, we obtain

$$
\begin{gathered}
M_{0} l_{0} \frac{\ddot{X}}{l}-M_{0} l_{0} \frac{\dot{X} \dot{l}}{l^{2}}=F^{\text {stat }}(X)-\beta M_{0} l_{0} \frac{\dot{X}}{l}+F_{e x}, \\
\alpha M_{0} l_{0} \frac{\ddot{l}}{l}+M_{0} l_{0} \frac{\dot{X}^{2}}{l^{2}}=K^{\text {int }}(l, \dot{l}, \dot{X})-\beta \alpha M_{0} l_{0} \frac{\dot{l}}{l}+K,
\end{gathered}
$$

where

$$
F_{e x}=\int_{-\infty}^{+\infty} d x f(t) \frac{\partial \phi}{\partial X}=-q f(t), \quad F^{s t a t}=0,
$$

$$
\begin{gathered}
K=\int_{-\infty}^{+\infty} d x f(t) \frac{\partial \phi}{\partial l}=0, \quad K^{i n t}=-\frac{\partial E}{\partial l}, \\
E=\frac{1}{2} \frac{l_{0}}{l} M_{0} \dot{X}^{2}+\frac{1}{2} \frac{l_{0}}{l} \alpha M_{0} \dot{l}^{2}+\frac{1}{2} M_{0}\left(\frac{l_{0}}{l}+\frac{l}{l_{0}}\right),
\end{gathered}
$$

with $\alpha=\left(\pi^{2}-6\right) / 12, \quad q=2$ and $M_{0}=4 /\left(3 l_{0}\right)$. Denoting $P(t) \equiv M_{0} l_{0} \dot{X} / l$, Eq. (13) can be written as

$$
\frac{d P}{d t}=-\beta P-q f(t)
$$

It is interesting to note that this equation may also be obtained by applying the McLaughlin-Scott procedure [6] with one collective variable only corresponding to the center of the kink. As shown in Ref. [18], for the sG kink dynamics this is an excellent description of the kink motion, and we have verified that it also describes the $\phi^{4}$ kink motion under ac forces away from the resonances we will find and discuss below. Its solution is given by

$$
\begin{aligned}
P(t)= & \frac{q \epsilon}{\left(\beta^{2}+\delta^{2}\right)}\left[\delta \cos \left(\delta t+\delta_{0}\right)-\beta \sin \left(\delta t+\delta_{0}\right)\right] \\
& +\exp (-\beta t)\left\{P(0)+\frac{q \epsilon}{\left(\beta^{2}+\delta^{2}\right)}\left[\beta \sin \left(\delta_{0}\right)\right.\right. \\
& \left.\left.-\delta \cos \left(\delta_{0}\right)\right]\right\} .
\end{aligned}
$$

From Eq. (14) the equation that holds for the width $l(t)$ is

$$
\alpha\left[\dot{l}^{2}-2 l \ddot{l}-2 \beta l \dot{l}\right]=\frac{l^{2}}{l_{0}^{2}}\left[1+\frac{P^{2}}{M_{0}^{2}}\right]-1 .
$$

Note that the term $P(t)^{2}$ in Eq. (20) involves two frequencies $\delta$ and $2 \delta$ when $\beta=0$, whereas for $\beta \neq 0$ and after some transient time the only frequency that remains is $2 \delta$. Furthermore, the term with frequency $\delta$ vanishes for $\beta=0$ and an appropriate choice of initial parameters. This equation represents a nonlinear, damped, and parametrically excited oscillator. To solve Eq. (20) we provide the change of variables, $l(t)=g^{2}(t)$, proposed in Ref. [19], which transforms the above equation into an Ermakov-type (or Pinney-type) equation (see Ref. [20], and references therein). Then the equation for $g(t)$ reads

$$
\begin{gathered}
\ddot{g}+\beta \dot{g}+\left[\left(\frac{\Omega}{2}\right)^{2}+\left(\frac{\Omega}{2 M_{0}}\right)^{2} P^{2}\right] g=\frac{1}{4 \alpha g^{3}}, \\
g(0)=\sqrt{l_{s}} \neq 0, \quad \dot{g}(0)=\frac{\dot{l}(0)}{2 \sqrt{l_{s}}},
\end{gathered}
$$

where $\Omega=1 / \sqrt{\alpha} l_{0}=1.2452$ is equal to the Rice frequency $\Omega_{R}=1 / \sqrt{\alpha} l_{s}$ in the case when the kink initially is at rest; this agrees within $1.7 \%$ with $\Omega_{i}=\sqrt{3 / 2}=1.2247$. We have not been able to solve Eq. (21) analytically, except when $\beta$ $=0$. Section III is devoted to a detailed analysis of that case; 
we will come back to the nonzero $\beta$ problem when discussing our numerical results in Sec. IV.

\section{UNDAMPED KINK: $\beta=0$}

When $\beta=0$, Eq. (21) reads

$$
\ddot{g}+\left[\left(\frac{\Omega}{2}\right)^{2}+\left(\frac{\Omega}{2 M_{0}}\right)^{2} P^{2}\right] g=\frac{1}{4 \alpha g^{3}},
$$

where $P(t)$ is given by $P(t)=\lambda+q \epsilon \cos \left(\delta t+\delta_{0}\right) / \delta$, with $\lambda$ $\equiv M_{0} \gamma_{0} u(0) / l_{s}-q \epsilon \cos \left(\delta_{0}\right) / \delta$. We thus see that the function $P(t)^{2}$ in Eq. (22) involves trigonometric functions with frequencies $\delta$ and $2 \delta$ if and only if $\lambda \neq 0$. Conversely, when $\lambda=0$ the only frequency that remains in the function $P(t)^{2}$ is $2 \delta$. Interestingly, we note that the relation $\lambda=0$ coincides with the condition for the oscillatory motion of the center of the kink, obtained by using the McLaughlin-Scott approach in the absence of dissipation [18]. The solution of Eq. (22) [21] is

$$
g(t)=\sqrt{v_{1}^{2}+\frac{1}{4 \alpha W^{2}} v_{2}^{2}},
$$

where $v_{1}(t)$ and $v_{2}(t)$ are two independent solutions of the linear part of Eq. (22). $W=\dot{v}_{1} v_{2}-\dot{v}_{2} v_{1}$ is the Wronskian, which in this case is a constant and can be calculated by the initial conditions for $v_{i}(i=1,2)$, which are $v_{1}(0)=\sqrt{l_{s}}$, $\dot{v}_{1}(0)=\dot{l}(0) /\left(2 \sqrt{l_{s}}\right), v_{2}(0)=0$, and $\dot{v}_{2}(0)=\mathrm{const} \neq 0$.

\section{A. $\lambda=0$ : resonance at $\delta \approx \Omega_{R} / 2$}

If one denotes $\tau=\delta t+\delta_{0}$, and sets $\lambda=0$ in the linear part of Eq. (22), after some manipulations we arrive at the following Mathieu equation for the $v_{i}$ functions:

$$
\begin{gathered}
v_{i}^{\prime \prime}+[a+2 \theta \cos (2 \tau)] v_{i}=0, \\
a=\left(\frac{\Omega}{2 \delta}\right)^{2}\left[1+\frac{q^{2} \epsilon^{2}}{2 \delta^{2} M_{0}^{2}}\right], \quad \theta=\left(\frac{\Omega}{2 \delta}\right)^{2} \frac{q^{2} \epsilon^{2}}{4 \delta^{2} M_{0}^{2}},
\end{gathered}
$$

where the prime denotes the derivative with respect to $\tau$. Note that the initial conditions for $v_{i}(\tau)$ become $v_{1}\left(\delta_{0}\right)$ $=\sqrt{l_{s}}, \quad v_{1}^{\prime}\left(\delta_{0}\right)=\dot{l}(0) /\left(2 \delta \sqrt{l_{s}}\right), \quad v_{2}\left(\delta_{0}\right)=0, \quad$ and $\quad v_{2}^{\prime}\left(\delta_{0}\right)$ $=\dot{v}(0) / \delta$. The solution of Eq. (24) (see Ref. [22]) for $v_{1}(\tau)$ and $v_{2}(\tau)$ can be expressed as a linear superposition of the two Mathieu functions $c e_{\nu}$ and $s e_{\nu}$ with a noninteger index $\nu$, i.e.,

$$
v_{i}(\tau)=A_{i} c e_{\nu}(\tau,-\theta)+B_{i} s e_{\nu}(\tau,-\theta), \quad i=1,2,
$$

where

$$
A_{i} \equiv \frac{\Delta_{A_{i}}}{\Delta}, \quad B_{i} \equiv \frac{\Delta_{B_{i}}}{\Delta},
$$

and

$$
\begin{gathered}
\Delta=c e_{\nu}\left(\delta_{0},-\theta\right) s e_{\nu}^{\prime}\left(\delta_{0},-\theta\right)-c e_{\nu}^{\prime}\left(\delta_{0},-\theta\right) s e_{\nu}\left(\delta_{0},-\theta\right), \\
\Delta_{A_{i}}=v_{i}\left(\delta_{0}\right) s e_{\nu}^{\prime}\left(\delta_{0},-\theta\right)-v_{i}^{\prime}\left(\delta_{0}\right) s e_{\nu}\left(\delta_{0},-\theta\right), \\
\Delta_{B_{i}}=v_{i}^{\prime}\left(\delta_{0}\right) c e_{\nu}\left(\delta_{0},-\theta\right)-v_{i}\left(\delta_{0}\right) c e_{\nu}^{\prime}\left(\delta_{0},-\theta\right),
\end{gathered}
$$

with the constraint (characteristic curve for Mathieu functions)

$$
a=\nu^{2}+\frac{1}{2\left(\nu^{2}-1\right)} \theta^{2}+O\left(\theta^{4}\right) .
$$

From Eqs. (23), (25), and (26), and taking into account that $\tau=\delta t+\delta_{0}$, we obtain that the kink width $l(t)$ is given by

$$
l(t)=g^{2}=v_{1}^{2}(t)+\frac{1}{4 \alpha W^{2}} v_{2}^{2}(t),
$$

$v_{i}(t)=A_{i} c e_{\nu}\left(\delta t+\delta_{0},-\theta\right)+B_{i} s e_{\nu}\left(\delta t+\delta_{0},-\theta\right), \quad i=1,2$,

where

$$
\begin{gathered}
A_{i} \equiv \frac{v_{i}(0) s e_{\nu}\left(\delta_{0},-\theta\right)-\dot{v}_{i}(0) s e_{\nu}\left(\delta_{0},-\theta\right)}{\left[c e_{\nu}\left(\delta_{0},-\theta\right) s e_{\nu}\left(\delta_{0},-\theta\right)-c e_{\nu}\left(\delta_{0},-\theta\right) s e_{\nu}\left(\delta_{0},-\theta\right)\right]}, \\
B_{i} \equiv-\frac{v_{i}(0) c e_{\nu}\left(\delta_{0},-\theta\right)-\dot{v}_{i}(0) c e_{\nu}\left(\delta_{0},-\theta\right)}{\left[c e_{\nu}\left(\delta_{0},-\theta\right) s e_{\nu}\left(\delta_{0},-\theta\right)-c e_{\nu}\left(\delta_{0},-\theta\right) s e_{\nu}\left(\delta_{0},-\theta\right)\right]},
\end{gathered}
$$

$$
W=-\sqrt{l_{s}} \dot{v}_{2}(0),
$$




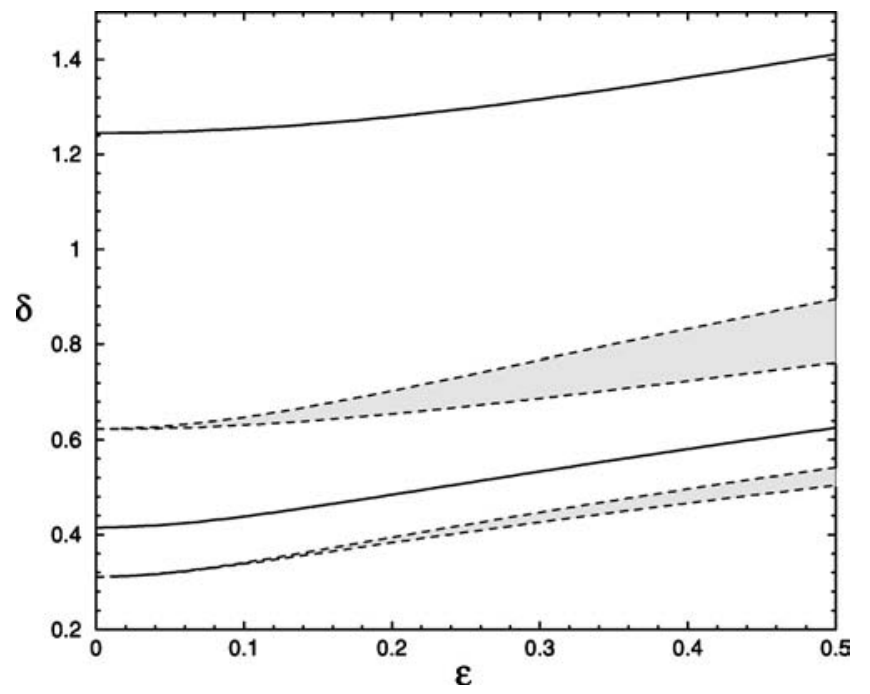

FIG. 1. Characteristic curves $\delta=\delta(\epsilon)$ corresponding to Eqs. (32) (upper solid line), (33), and (34) (upper dashed lines) for zero initial kink velocity and $\delta_{0}=\pi / 2$. The lower solid line is related with solutions (28) $-(31)$ when $\nu=3 / 2$. The lower dashed lines are the characteristic curves of the integer Mathieu functions with $n$ $=2$. Shadowed regions represent unstable solutions of Eq. (24).

and the characteristic curve [Eq. (27)] for our initial parameters can be written up to order $\epsilon^{2}$ as

$$
\delta=\frac{\Omega_{R}}{2 \nu}-\frac{q^{2} \nu \cos \left(2 \delta_{0}\right)}{2 M_{0}^{2} \gamma_{0}^{2} \Omega_{R}} \epsilon^{2}+O\left(\epsilon^{4}\right) .
$$

To obtain a better approximation, we need to take into account more terms of the above series, so in Fig. 1 we plot, as solid lines, the characteristic curves obtained numerically with MATHEMATICA 3.0 [23], for $\nu=1 / 2$ and $3 / 2$ when $u(0)$ $=0$ and $\delta_{0}=\pi / 2$. Note that when $\nu=m+p / s$ is rational, with $m$ an integer number and $p / s$ a rational fraction $(0$ $<p / s<1), v_{1}(t)$ or $v_{2}(t)$ are $2 \pi s$ periodic functions if $p$ is odd, and $\pi s$-periodic functions if $p$ is even, however, for irrational $\nu$ both functions will be nonperiodic, but bounded, solutions [22].

If we know $l(t)$, then from the solution $P(t)$ [Eq. (19)] we can calculate the velocity $\dot{X}=P(t) l(t) /\left(M_{0} l_{0}\right)$ for the kink center. Since the momentum $P(t)$ is a periodic function and the kink width $l(t)$ at least is a bounded function, $\dot{X}$ is bounded as well. For instance, if we take $\nu=1 / 2$ in Eq. (32), the frequency of the external force,

$$
\delta=\Omega_{R}-\frac{q^{2} \cos \left(2 \delta_{0}\right)}{4 M_{0}^{2} \gamma_{0}^{2} \Omega_{R}} \epsilon^{2}+O\left(\epsilon^{4}\right)
$$

is very close to $\Omega_{R} \approx \Omega_{i}$ if $\epsilon \ll 1$ (see Fig. 1), and $l(t)$ is a function of the square of the $4 \pi / \delta$-periodic solutions $s e_{1 / 2}$ and $c e_{1 / 2}$, respectively, thus the velocity of the kink center, $\dot{X}(t)$, and the energy $E(t)$ will also be bounded functions for $\delta=\delta(\epsilon)$.

If we try to find, e.g., $2 \pi$-periodic solutions of Eq. (24), we obtain that in this case the two independent solutions are related to the integer Mathieu functions $s e_{1}$ and $c e_{1}$. However, these two solutions appear when the characteristic curve $a(\theta)$ is

$$
a=1+\theta-\frac{\theta^{2}}{8}-\frac{\theta^{3}}{64}-\frac{\theta^{4}}{1536}+\cdots
$$

for the even Mathieu function $c e_{1}$, and

$$
a=1-\theta-\frac{\theta^{2}}{8}+\frac{\theta^{3}}{64}-\frac{\theta^{4}}{1536}+\cdots
$$

for the odd Mathieu function $s e_{1}$. Since the values of $a$ are different for each of the function $s e_{1}$ and $c e_{1}$, these functions are not solutions of the same equation except when $\theta$ $=0$. Although $s e_{1}$ and $c e_{1}$ are not solutions of Eq. (24) [recall that in order to find $l(t)$ we need to calculate two independent solutions of Eq. (24)], the characteristic curves of these solutions separate the unstable and stable solutions of the Mathieu equation (24), and can be rewritten as

$$
\delta_{-}=\frac{\Omega_{R}}{2}-\left[\frac{q^{2} \cos \left(2 \delta_{0}\right)}{2 M_{0}^{2} \gamma_{0}^{2} \Omega_{R}}+\frac{q^{2}}{4 M_{0}^{2} \gamma_{0}^{2} \Omega_{R}}\right] \epsilon^{2}
$$

and

$$
\delta_{+}=\frac{\Omega_{R}}{2}-\left[\frac{q^{2} \cos \left(2 \delta_{0}\right)}{2 M_{0}^{2} \gamma_{0}^{2} \Omega_{R}}-\frac{q^{2}}{4 M_{0}^{2} \gamma_{0}^{2} \Omega_{R}}\right] \epsilon^{2},
$$

respectively. Interestingly, we note that the width of the unbounded region,

$$
\Delta \delta \equiv \delta_{+}-\delta_{-}=\frac{q^{2} \epsilon^{2}}{2 M_{0}^{2} \gamma_{0}^{2} \Omega_{R}},
$$

decreases when the initial velocity increases, and consequently we will focus on the case $u(0)=0$ in our numerical simulations below, as the resonance is then easier to observe. The curves $\delta_{+}$and $\delta_{-}$[Eqs. (33) and (34)] are plotted in Fig. 1 for zero initial velocity and $\delta_{0}=\pi / 2$, where the shadowed region represents the region where $l(t)$ is unbounded. Analogously, we can obtain other characteristic curves, related either to integer Mathieu functions $s e_{n}$ and $c e_{n}(n \in \mathrm{N})$, or to noninteger Mathieu functions $s e_{\nu}$ and $c e_{\nu}$. In view of this, the above analytical results lead us to expect that if $\delta$ is close to $\Omega_{R} \approx \Omega_{i}, l(t), u(t)$, and the energy should be oscillatory (or, at least, bounded) functions; whereas if $\delta \approx \Omega_{R} / 2$ $\approx \Omega_{i} / 2$ the kink width $l(t)$ increases indefinitely, and since the energy and velocity are proportional to $l(t)$ [see Eqs. (19) and (17)] we should observe that these functions increase with time as well.

\section{B. $\lambda \neq 0$ : resonances at $\delta \approx \Omega_{R} / 2, \Omega_{R}$}

When $\lambda \neq 0$, the linear part of Eq. (22) becomes, in a manner similar to the procedure leading to Eq. (24), a more general Mathieu equation [24], namely,

$$
g^{\prime \prime}+\left[b+2 \theta_{1} \epsilon^{2} \cos (2 \tau)+2 \theta_{2} \epsilon^{2} \cos (4 \tau)\right] g=0,
$$

where 


$$
\begin{gathered}
b=\left(\frac{\Omega}{\delta}\right)^{2}\left[1+\frac{q^{2} \epsilon^{2}}{2 \delta^{2} M_{0}^{2}}+\frac{\lambda^{2}}{M_{0}^{2}}\right], \\
\theta_{1}=\left(\frac{\Omega}{\delta}\right)^{2} \frac{\lambda q^{2}}{\delta^{2} M_{0}^{2}}, \quad \theta_{2}=\left(\frac{\Omega}{\delta}\right)^{2} \frac{q^{2}}{4 \delta^{2} M_{0}^{2}},
\end{gathered}
$$

and the prime denotes the derivative with respect to $\tau=(\delta t$ $\left.+\delta_{0}\right) / 2$. According to Floquet theory [25], Eq. (35) has normal solutions of the form $g=\exp (\sigma \tau) \Phi(\tau)$, where $\Phi(\tau)$ is a $\pi$-periodic function and $\sigma$ is the characteristic exponent. Expanding $\Phi(\tau)$ in a Fourier series, the function $g$ can be rewritten as $g=\sum_{n=-\infty}^{+\infty} \Phi_{n} \exp [(\sigma+2 n i) \tau]$, where $\Phi_{n}$ are the coefficients of the above series. Therefore, the transition curves separating stability from instability correspond to $\sigma$ $=0(\pi$-periodic solutions $)$ and $\sigma=i$ ( $2 \pi$-periodic solutions $)$ [26]. Now we will apply the method of strained parameters [25] to determine the values of $b, \theta_{1}$, and $\theta_{2}$ corresponding to these values of the characteristic exponent. First we assume that the solutions of Eq. (35), which have period $\pi$ or $2 \pi$, and the transition curves $b=b(\epsilon)$ can be written in the form of perturbation expansions as

$$
\begin{gathered}
g(\tau)=g_{0}+\epsilon^{2} g_{1}+\epsilon^{4} g_{2}+O\left(\epsilon^{6}\right), \\
b=b_{0}+\epsilon^{2} b_{1}+\epsilon^{4} b_{2}+O\left(\epsilon^{6}\right) .
\end{gathered}
$$

Second, substituting Eq. (36) into Eq. (35) and equating the coefficients of the same order of $\epsilon$ to zero, we obtain

$$
\begin{gathered}
\mathcal{L} g_{0}=0, \\
\mathcal{L} g_{1}=-b_{1} g_{0}-2 \theta_{1} \cos (2 \tau) g_{0}-2 \theta_{2} \cos (4 \tau) g_{0}, \\
\mathcal{L} g_{2}=-b_{1} g_{1}-b_{2} g_{0}-2 \theta_{1} \cos (2 \tau) g_{1}-2 \theta_{2} \cos (4 \tau) g_{1},
\end{gathered}
$$

where $\mathcal{L}$ represent the second-order linear differential operator $\mathcal{L}=\left(d^{2} / d \tau^{2}\right)+b_{0}$. The solution of Eq. (37) is

$$
g_{0}=A \cos \left(\sqrt{b_{0}} \tau\right)+B \sin \left(\sqrt{b_{0}} \tau\right),
$$

where $b_{0}=4 n^{2}$ for the $\pi$-periodic solutions and $b_{0}=(2 n$ $-1)^{2}$ for the $2 \pi$-periodic solutions; $n$ is an integer number, and $A$ and $B$ are constants.

For the $\pi$-periodic solutions we will analyze the case $n$ $=1$, corresponding to $b_{0}=4$; we note that the case $n=0$ is not possible due to the definition of our parameter $b$. When $b_{0}=4, g_{0}=A \cos (2 \tau)+B \sin (2 \tau)$. For $g_{1}$ to be a $\pi$-periodic function it is necessary to eliminate the secular terms in Eq. (38), imposing either $b_{1}=\theta_{2}$ and $A=0$ or $b_{1}=-\theta_{2}$ and $B$ $=0$. Then the transition curves corresponding to the solutions

$$
g_{+}=B \sin (2 \tau)+B \epsilon^{2}\left[\frac{\theta_{1}}{20} \sin (4 \tau)+\frac{\theta_{2}}{32} \sin (6 \tau)\right]
$$

and

$$
g_{-}=A \cos (2 \tau)+A \epsilon^{2}\left[\frac{-\theta_{1}}{4}+\frac{\theta_{1}}{12} \cos (4 \tau)+\frac{\theta_{2}}{32} \cos (6 \tau)\right]
$$

can be shown to be

$$
b=4 \pm \epsilon^{2} \theta_{2},
$$

or, alternatively,

$$
\begin{gathered}
\delta_{+}=\frac{\Omega_{R}}{2}-\frac{q u(0) \cos \left(\delta_{0}\right)}{M_{0} \gamma_{0}} \epsilon+\left[\frac{3 q^{2}}{4 M_{0}^{2} \gamma_{0}^{2} \Omega_{R}}+\frac{q^{2} \cos \left(2 \delta_{0}\right)}{2 M_{0}^{2} \gamma_{0}^{2} \Omega_{R}}\right] \epsilon^{2}, \\
\delta_{-}=\frac{\Omega_{R}}{2}-\frac{q u(0) \cos \left(\delta_{0}\right)}{M_{0} \gamma_{0}} \epsilon+\left[\frac{5 q^{2}}{4 M_{0}^{2} \gamma_{0}^{2} \Omega_{R}}+\frac{q^{2} \cos \left(2 \delta_{0}\right)}{2 M_{0}^{2} \gamma_{0}^{2} \Omega_{R}}\right] \epsilon^{2},
\end{gathered}
$$

respectively. Since these curves start from $\epsilon=0$ and $b=4$, we are analyzing the transition curves when the frequency of the ac force is close to half the value of $\Omega_{R}$. Hence the resonance at $\delta \approx \Omega_{i} / 2 \approx \Omega_{R} / 2$ appears much as in the case $\lambda$ $=0$, even when $\lambda \neq 0$. Let us remark that, for a given $\epsilon$, the distance between these two curves $\delta=\delta(\epsilon)$ is

$$
\frac{q^{2} \epsilon^{2}}{2 M_{0}^{2} \gamma_{0}^{2} \Omega_{R}}
$$

so when the velocity increases, the unstable region is narrower, as we found above in the case when $\lambda=0$.

The other transition curves are related to the $2 \pi$-periodic solution of Eq. (37), $g_{0}=A \cos (\tau)+B \sin (\tau)$, which appears when $b_{0}=1 \quad(n=1)$. In this case $g_{1}$ will be a periodic function if $b_{1}=\theta_{1}$ and $A=0$ or $b_{1}=-\theta_{1}$ and $B=0$. Hence the transition curves starting from $b=1$ are

$$
b=1 \pm \epsilon^{2} \theta_{1},
$$

or, equivalently,

$$
\begin{aligned}
\delta_{\mp}= & \Omega_{R}-\frac{q u(0) \cos \left(\delta_{0}\right)}{M_{0} \gamma_{0}} \epsilon+\left[\frac{q^{2}}{2 M_{0}^{2} \gamma_{0}^{2} \Omega_{R}}+\frac{q^{2} \cos \left(2 \delta_{0}\right)}{4 M_{0}^{2} \gamma_{0}^{2} \Omega_{R}}\right] \epsilon^{2} \\
& \mp \frac{q^{2} \lambda}{2 M_{0}^{2} \gamma_{0}^{2} \Omega_{R}} \epsilon^{2},
\end{aligned}
$$

and correspond to the solutions

$$
\begin{gathered}
g_{-}=B \sin (\tau)+B \epsilon^{2}\left[\frac{\theta_{1}-\theta_{2}}{8} \sin (3 \tau)+\frac{\theta_{2}}{24} \sin (5 \tau)\right], \\
g_{+}=A \cos (\tau)+A \epsilon^{2}\left[\frac{\theta_{1}+\theta_{2}}{8} \cos (3 \tau)+\frac{\theta_{2}}{24} \cos (5 \tau)\right],
\end{gathered}
$$

respectively. These solutions, and their corresponding characteristic curves, are related to the driving frequency $\delta$ close to $\Omega_{R}$. Finally, in this case we have

$$
\Delta \delta \equiv \delta_{+}-\delta_{-}=\frac{q^{2} \lambda \epsilon^{2}}{M_{0}^{2} \gamma_{0}^{2} \Omega_{R}}
$$

which means, as above, that for a fixed $\lambda, \Delta \delta$ decreases when $u(0)$ is increased. 


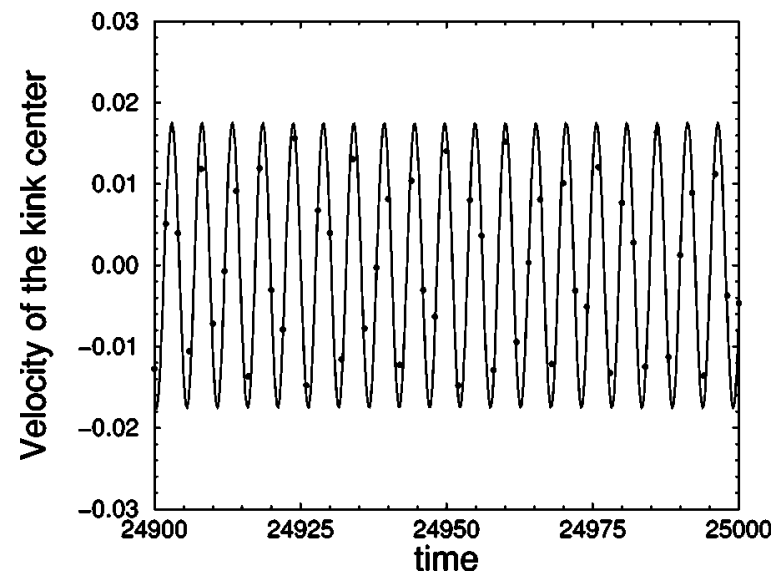

FIG. 2. Verification of the collective coordinate method in the absence of damping. The solid line was obtained from Eqs. (18) and (20), and represents the values of the velocity of the kink center as given by the collective coordinate approach; the points are the velocities of the center of the kink from the numerical simulations of Eqs. (2) and (3) by using the Strauss-Vázquez scheme, starting from a kink at rest and with force $f(t)=0.01 \sin (1.21 t+\pi / 2)$. The velocity function is represented only from $t=24900$ to the final time $t=25000$, but the same behavior of this function is observed during the whole run.

In summary, we have found here that when $\lambda \neq 0$ and we drive the system with a frequency close to $\Omega_{R}$, the solutions will be unstable in the region between curves $\delta_{+}$and $\delta_{-}$. Note that for $\lambda=0$ we recover the previous results, i.e., there are no resonances at $\delta \approx \Omega_{R}$.

\section{NUMERICAL VERIFICATION}

The results we obtained in Sec. III were derived within the collective coordinate assumption that we can describe all the kink dynamics by the two variables $X(t)$ and $l(t)$, all other degrees of freedom being negligible. As there is no way to know a priori that this is indeed the case, we have to verify this hypothesis by numerical simulations of the full partial differential equation. Furthermore, we have not been able to solve the collective coordinate equations for the damped $(\beta \neq 0)$ case; it is reasonable to expect that in this situation phenomena similar to those found for the undamped case will arise, but insofar as it has not been checked, this assertion remains a conjecture.

In view of the above considerations, we have computed the numerical solution of the partial differential equations (2) and (3) by using the Strauss-Vázquez scheme [27] and choosing a total length for our numerical system of $L$ $=400$, with steps $\Delta t=0.01, \Delta x=0.1$, free boundary conditions, and simulating up to a final time equal to 25000 with a kink at rest as initial condition. In addition, we have fixed the amplitude $\epsilon=0.01$ and the phase $\delta_{0}=\pi / 2$ of the ac force, and then changed the value of the driving frequency $\delta$ in order to see the resonances. We note that there are many other parameters we could change, such as the initial velocity, the driving amplitude, or the driving phase, but as our main goal in this section is to assert the validity of the general analytical results obtained above we prefer to concentrate on a few cases as mentioned. In all our simulations we have chosen the initial parameters in such a way that $\lambda$

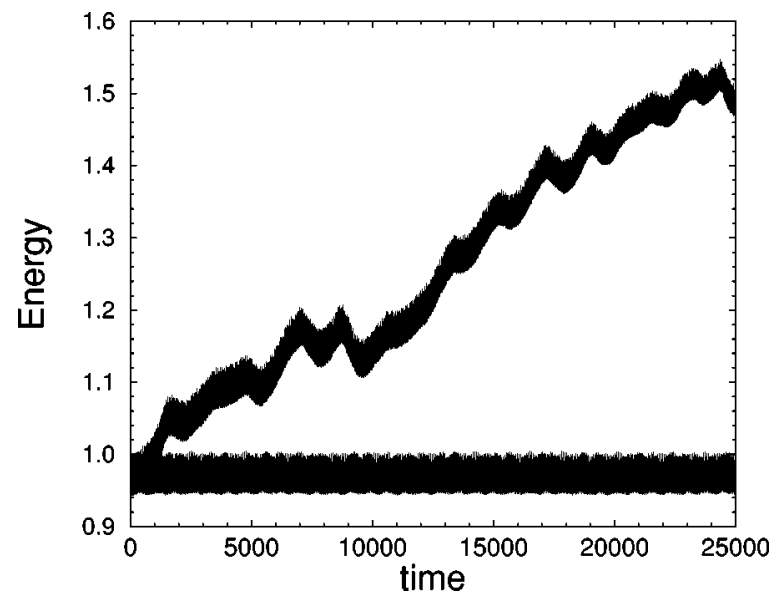

FIG. 3. Kink energy when the frequency of the driving force $\delta$ is close to $\Omega_{i} / 2$. The parameters of the numerical simulations of the full partial differential equations, Eqs. (2) and (3), are $\beta=0, \epsilon$ $=0.01, \delta_{0}=\pi / 2, u(0)=0, \delta=0.6104$ (upper curve, resonantly increasing energy), $\delta=0.608$ (lower curve, nonresonant oscillations), $L=400, \Delta x=0.1$, and $\Delta t=0.01$. Note that half of the Rice frequency is $\Omega_{R} / 2=0.6226$, and half of the frequency of the internal mode is $\Omega_{i} / 2=0.6124$.

$=0$, because when $\lambda \neq 0$ the kink moves to the right or to the left, and then for large times the kink will leave our finite system. For the aforementioned values of initial parameters the width of the resonance region $\Delta \delta=\delta_{+}-\delta_{-}$, predicted at $\delta \approx \Omega_{R} / 2 \approx \Omega_{i} / 2$ in Sec, III A, [see Eqs. (33) and (34)], is of the order of $10^{-4}$. For this reason we have explored the regions around $\Omega_{i}$ and $\Omega_{i} / 2$ in an interval of that order. Finally, we mention that with the Strauss-Vázquez method one can very accurately compute the position and velocity of the kink center using the integrals of energy and momentum [28]; thus this is a good numerical method in order to compare with our analytical predictions.

First of all, in order to verify the results obtained by means of the GTWA with two independent collective coordinates, the position and width of the kink, we have studied the region around $\delta \simeq \Omega_{i}=1.2247$, i.e., the driving frequency for which we do not expect any resonances when $\lambda=0$; hence the width and the velocity should at least be bounded functions. We have verified that this prediction is in excellent agreement with the numerical simulations, an example of which is shown in Fig. 2. In this plot, we can see that the velocity is indeed an oscillatory function, and even for large times our theoretical approach correctly describes the evolution of the velocity of the center of mass of the kink. Other values close to $\delta=1.21$ behave very much like the presented example.

The next test of our analytical results of course has to be the existence of a resonance near $\Omega_{i} / 2$. For frequencies $\delta$ around $\Omega_{R} / 2$, our collective variable approach has predicted that the width $l(t)$ increases unboundedly, and hence the velocity and energy should increase as well. Again, the prediction is fulfilled, but for $\Omega_{i} / 2$ instead of $\Omega_{R} / 2$ : In the numerical simulations we have observed that in this case a resonance takes place when $\delta \simeq \Omega_{i} / 2=0.6124$. As a specific example, the results for the kink energy for $\delta=0.6104$ and 0.608 are plotted in Fig. 3, clearly showing a resonant increase of the energy in the former case and an oscillatory 

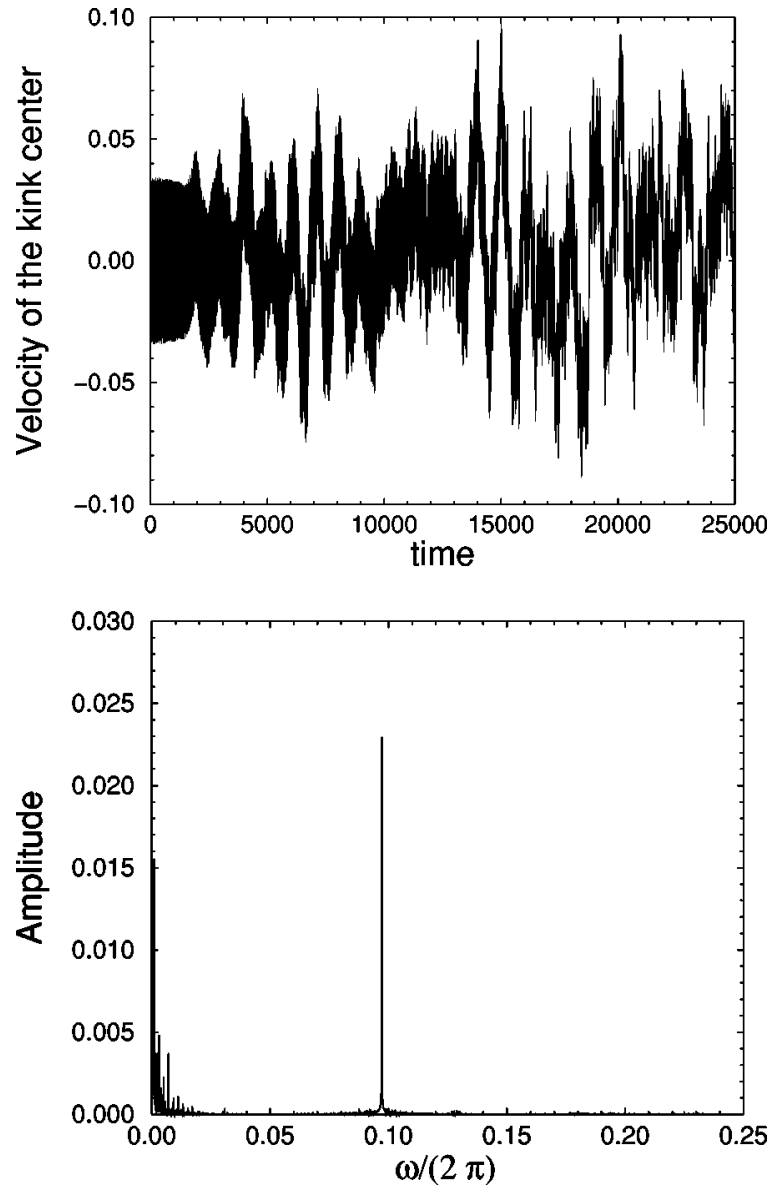

FIG. 4. Upper panel: Velocity of the kink center computed from the numerical solution of the partial differential equation when the frequency of the driving is close to half of the internal mode frequency $(\delta=0.6104), \beta=0, \epsilon=0.01, \delta_{0}=\pi / 2$, and $u(0)=0$. After some time the velocity function departs from its oscillatory behavior, which was transiently exhibited at the beginning of the run, and develops chaotic features. Lower panel: discrete Fourier transform of the signal in the upper panel.

behavior in the latter. Interestingly, at resonance the velocity also does not have an oscillatory behavior as depicted in Fig. 4 , in contrast to the behavior off-resonance we found in Fig. 2 for $\delta=1.21$. Such a nonperiodic evolution of the kink velocity implies that the kink motion is chaotic, as we first found in Ref. [15] and eventually the kink begins emitting radiation. As we discussed in Ref. [15], we believe that this phenomenon has its origin in the energy transfer from the kink internal mode to the rest of modes in the system, a mechanism already demonstrated in Refs. [12,14]; intuitively, the reason for this is that the collective coordinate prediction that the kink width grows without limit cannot be physically true due to the very nature of the kink, and hence, when the internal mode excitation reaches large values the energy ends up being transferred to the rest of the available modes (translation and radiation).

After checking our analytical results for the undamped case, we now have to turn to the damped $(\beta \neq 0)$ dynamics, in order to find out whether the same phenomena arise there. Simulations for the damped case show that the energy of the system also increases when resonance takes place (see Fig. $5)$, but in this case the energy is bounded due to the dissipa-

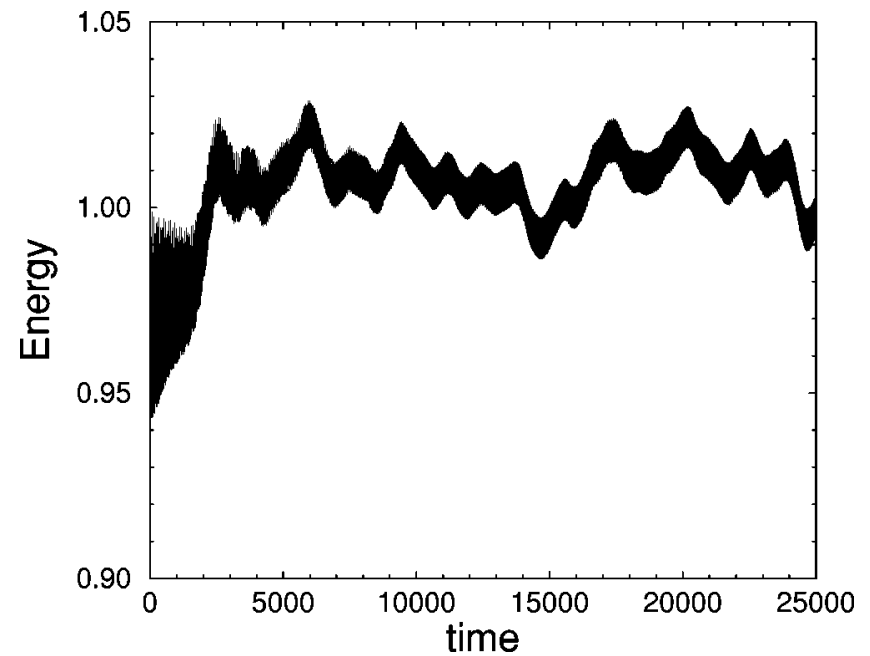

FIG. 5. Resonance in the presence of damping: kink energy at a frequency $\delta=0.6103$ obtained from numerical simulations of Eqs. (2) and (3), with $\epsilon=0.01, \beta=0.001, u(0)=0$, and $\delta_{0}=\pi / 2$.

tion, reaches an asymptotic value, and thus does not increase indefinitely as in the absence of damping. For a comparison of both situations we have computed the mean energy in the time interval $10000<t<25000$ with and without damping, and our results are summarized in Figs. 6 and 7. For $\beta$ $=0.001$ (see the points on the solid line in Fig. 6) we have found that the energy increases at $\delta=0.6103$, whereas for $\beta=0$ (see the points on the dashed line in Fig. 6) the resonance frequency is $\delta \approx 0.6102$.

As mentioned above, the numerical solutions of the full partial differential equation show that the resonance at $\delta$ $\approx \Omega_{i} / 2$ also takes place for $\beta \neq 0$, whereas when $\delta \approx \Omega_{i}$ (see Fig. 7) the resonance disappears. Let us recall that in all our simulations we have chosen $u(0)=0$ (and hence the resonant region should be the widest possible one) and $\delta_{0}$ $=\pi / 2$ in such a way that $\lambda=0$. In the case when $\lambda$ vanishes we cannot expect any resonances at $\delta \approx \Omega_{i}$. Nevertheless, in Fig. 7 it is clear that the energy increases weakly in this region. We believe that this maximum of the energy is prob-

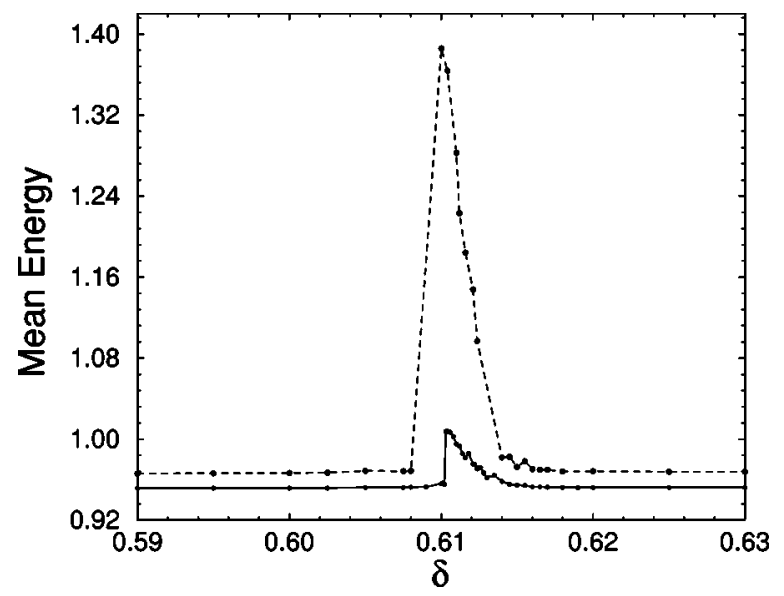

FIG. 6. Resonance at $\Omega_{i} / 2$. Mean energy of the system, computed by averaging the energy in the numerical simulations of the partial differential equations for $t>10000$, with the final time equal to $25000 ; \epsilon=0.01, \delta_{0}=\pi / 2$, and $u(0)=0$. Dashed line: undamped case, $\beta=0$; solid line: damped case, $\beta=0.001$. 


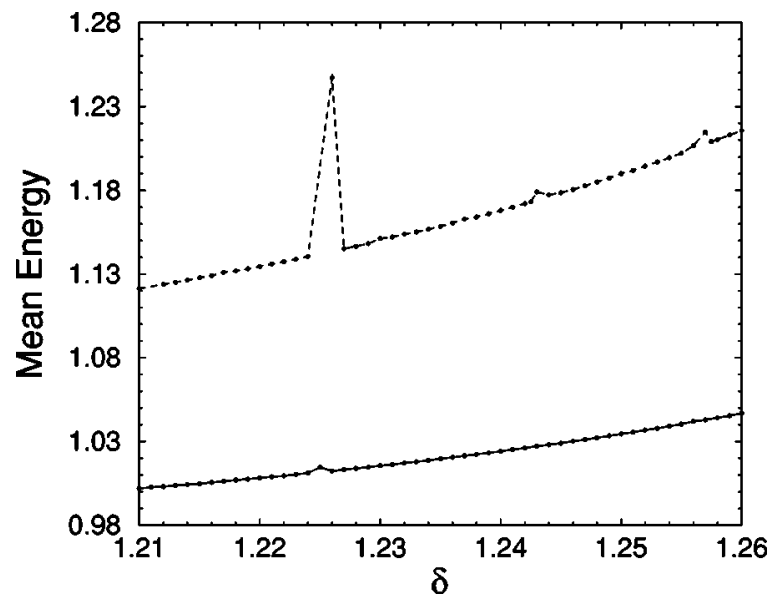

FIG. 7. Resonance at $\Omega_{i}$. Mean energy of the system, computed averaging the energy in the numerical simulations of the partial differential equations for $t>10000$, with the final time equal to $25000 ; \epsilon=0.01, \delta_{0}=\pi / 2, u(0)=0, \beta=0$ (dashed line), and $\beta$ $=0.001$ (solid line).

ably due to the fact that the condition for the suppression of the resonance at $\delta \approx \Omega_{i}$ is extremely difficult to fulfill numerically. On the other hand, it is also possible that this condition, which has been obtained within the collective coordinate framework, is only approximately true, and therefore when we choose $\lambda=0$ we are beginning with an initial condition close to the one needed for a complete suppression of the resonance but not exactly there; hence we would still see the small bump in Fig. 7. Another possible reason for this peak is that it is possible that when $\lambda=0$ at $\delta \approx \Omega_{i} \approx \Omega_{R}$ a resonance appears in the next order corrections (see the work of Segur [29], in which the frequency $2 \Omega_{i}$ arises, and could then be parametrically excited by $\delta=\Omega_{i}$ ). Were this the case, this "weaker resonance," that completely disappears for $\beta \neq 0$ (see the solid line in Fig. 7), is the resonance that we can expect either for $\lambda \approx 0$ in the partial differential equation or for $\lambda=0$ and large enough times.

\section{CONCLUSIONS}

We have shown analytically and numerically that the internal mode of the $\phi^{4}$ model can be excited if we drive the system with an ac force of a frequency close to $1 / 2$ of the internal mode frequency. This is a very surprising result, as the driving we apply to the system is not parametric. At resonance, as a consequence of the increment of the energy of the system, the kink initially at rest begins to move chaotically [15] and also begins to radiate, i.e., the energy is transferred from the internal mode to the translational and radiational ones. The chaotic motion is confirmed by noting that, when the internal mode is excited at $\delta \approx \Omega_{i} / 2$, in the discrete Fourier transform of the kink's velocity (see Fig. 4) we see some frequencies in the low frequency part of the spectrum aside from the frequency of the ac force, $\delta$. What is more important, we have presented a full analysis of the collective coordinate theory for this problem, whose validity has undoubtedly been confirmed by numerical simulations of the full partial differential equation. In particular, in the absence of damping we have been able to find all the resonances, as well as their dependence on the kink initial con- ditions, analytically. On the other hand, we have also shown that the resonance at $\delta \approx \Omega_{i}$ can be suppressed if we add a small damping to the system, or by an appropriate choice of initial parameters of the ac force. We have verified in our simulations that for $\delta \approx \Omega_{i}$ the lower radiational modes are excited; for this reason the energy in Fig. 7 increases when $\delta$ increases. Of course, this excitation of the lowest phonons cannot be explained within the present collective coordinate theory; a much more involved approach including phonon effects (as in Ref. [30] for the sG model) would probably account for that resonance.

Beyond the application of our results to the $\phi^{4}$ kink dynamics, we believe that the same phenomenology will arise in other systems for which internal modes are present (as, for example, in the double sG equation [31]). The analysis we presented can be straightforwardly extended to this system, and it is quite likely that similar resonance effects will arise, as well as in other models with the same feature of internal modes. A related interesting question concerns the applicability of these results to the sG equation. As we mentioned in Sec. I, our calculation applies directly to the $\mathrm{sG}$ equation, but in this system the kinks do not possess internal modes. Therefore, the identification $\Omega_{i} \approx \Omega_{R}$ we made for the $\phi^{4}$ kink is no longer available, and, what is worse, the putative $\Omega_{R}$ lies within the phonon band. However, it has been reported that close to $\Omega_{R}$ sG kinks might exhibit a quasimode [16] with a very long life. If such a mode indeed exists, which is still in doubt since no other reports of its existence have been published, it could be found, as we have done in this paper, by looking at resonances of half its frequency, which would be outside the phonon band. Work along these lines is in progress [32].

\section{ACKNOWLEDGMENTS}

We thank Yuri Gaididei, Francisco Domínguez-Adame, and José Cuesta for discussions. Work at GISC (Leganés) was supported by DGESIC (Spain) Grant No. PB96-0119. Travel between Bayreuth and Madrid was supported by "Acciones Integradas Hispano-Alemanas," a joint program of DAAD (Az. 314-AI) and DGESIC.

\section{APPENDIX A: EQUIVALENCE OF COLLECTIVE COORDINATE METHODS}

In this appendix we demonstrate that the GTWA, the method we used in Sec. II to obtain the collective coordinate equations, is equivalent to using the variation of the momentum and the energy of system (1). First of all, from Eqs. (7) and (10) we can obtain the variation of the momentum and energy of the perturbed $\phi^{4}$ equation. Combining the first three terms of Eq. (7), and doing some straightforward transformations the equation for the momentum can be recast in the forms

$$
\begin{gathered}
\frac{d P}{d t}=-\int_{-\infty}^{+\infty} d x \frac{\partial \mathcal{H}}{\partial X}+\int_{-\infty}^{+\infty} d x F\left(x, t, \phi, \phi_{t}, \ldots\right) \frac{\partial \phi}{\partial X}, \\
P(t)=-\int_{-\infty}^{+\infty} d x \phi_{x} \phi_{t} .
\end{gathered}
$$


Furthermore, if instead of Eq. (10) we add Eq. (7), rewritten as

$$
\begin{gathered}
\int_{-\infty}^{+\infty} d x \frac{\partial \phi}{\partial X} \frac{\partial \psi}{\partial \dot{X}} \ddot{X}+\int_{-\infty}^{+\infty} d x[\phi, \psi] \dot{l}+\int_{-\infty}^{+\infty} d x \frac{\partial \phi}{\partial X} \frac{\partial \psi}{\partial \dot{l}} \ddot{l} \\
-F^{s t a t}(X)-\int_{-\infty}^{+\infty} d x F\left(x, t, \phi, \phi_{t}, \ldots\right) \frac{\partial \phi}{\partial X}=0,
\end{gathered}
$$

multiply this equation by $\dot{X}$, and the expression obtained from Eq. (10) by $\dot{l}$, we conclude that Eq. (10) is equivalent to

$$
\begin{gathered}
\frac{d H}{d t}=\int_{-\infty}^{+\infty} d x F\left(x, t, \phi, \phi_{t}, \ldots\right) \frac{d \phi}{d t}, \\
H(t)=\int_{-\infty}^{+\infty} d x\left\{\frac{\phi_{x}^{2}}{2}+\frac{\phi_{t}^{2}}{2}+U(\phi)\right\} .
\end{gathered}
$$

This implies that when we apply the GTWA in those systems we are, in fact, varying the momentum,

$$
\frac{d P}{d t}=-\frac{d}{d t} \int_{-\infty}^{+\infty} d x \phi_{x} \phi_{t}=-\int_{-\infty}^{+\infty} d x\left[\phi_{x t} \phi_{t}-\phi_{x} \phi_{t t}\right],
$$

and the energy,

$$
\begin{aligned}
\frac{d H}{d t} & =\frac{d}{d t} \int_{-\infty}^{+\infty} d x\left\{\frac{1}{2} \phi_{x}^{2}+\frac{1}{2} \phi_{t}^{2}+U(\phi)\right\} \\
& =\int_{-\infty}^{+\infty} d x\left\{\phi_{x} \phi_{x x}+\phi_{t} \phi_{t t}+U^{\prime}(\phi) \phi_{t}\right\},
\end{aligned}
$$

where

$$
\begin{gathered}
\phi(x, t)=\phi[x-X(t), l(t)], \\
\phi_{t} \equiv \psi(x, t)=\psi[x-X(t), l(t), \dot{X}(t), \dot{l}(t)] .
\end{gathered}
$$

Moreover, we can also start from Eqs. (A3), (A4), and (A5), and obtain Eqs. (7) and (10). Doing this we only need to take into account that there are at least two different ways of transforming the above integrals: in one of them, one substitutes Eq. (A5) into Eqs. (A3) and (A4), so that

$$
\begin{gathered}
\frac{d P}{d t}=\int_{-\infty}^{+\infty} d x\left\{\frac{\partial \phi}{\partial X} \frac{\partial \psi}{\partial \dot{X}} \ddot{X}+[\phi, \psi] \dot{l}+\frac{\partial \phi}{\partial X} \frac{\partial \psi}{\partial \dot{l}} \ddot{l}\right\}, \\
\frac{d H}{d t}=\dot{X} \int_{-\infty}^{+\infty} d x\left\{\frac{\partial \mathcal{H}}{\partial X}+\frac{\partial \phi}{\partial X} \frac{\partial \psi}{\partial \dot{X}} \ddot{X}+[\phi, \psi] \dot{l}+\frac{\partial \phi}{\partial X} \frac{\partial \psi}{\partial \dot{l}} \ddot{l}\right\} \\
+i \int_{-\infty}^{+\infty} d x\left\{[\psi, \phi] \dot{X}+\frac{\partial \phi}{\partial l} \frac{\partial \psi}{\partial \dot{X}} \ddot{X}+\frac{\partial \phi}{\partial l} \frac{\partial \psi}{\partial \dot{l}} \ddot{l}\right\},
\end{gathered}
$$

whereas, in the other one, we first substitute the systems of equation (2) and (3) into Eqs. (A3) and (A4), and then assume that $\phi(x, t)$ and $\psi(x, t)$ satisfy Eq. (A5). Thus

$$
\frac{d P}{d t}=-\int_{-\infty}^{+\infty} d x \frac{\partial \mathcal{H}}{\partial X}+\int_{-\infty}^{+\infty} d x F\left(x, t, \phi, \phi_{t}, \ldots\right) \frac{\partial \phi}{\partial X},
$$

$$
\begin{aligned}
\frac{d H}{d t}= & \dot{X} \int_{-\infty}^{+\infty} d x F\left(x, t, \phi, \phi_{t}, \ldots\right) \frac{\partial \phi}{\partial X} \\
& +i \int_{-\infty}^{+\infty} d x F\left(x, t, \phi, \phi_{t}, \ldots\right) \frac{\partial \phi}{\partial l} .
\end{aligned}
$$

Eqs. (A6) and (A8) and Eqs. (A7) and (A9), respectively, we find that Eqs. (A3) and (A4) become Eqs. (7) and (10), respectively.

Let us note that the connection between the GTWA and the variation of the momentum and energy gives a physical interpretation of such a technique, and furthermore, this second method leads more directly to formulas for $X(t)$ and $l(t)$. Furthermore, if the solution of Eqs. (2) and (3) for $F\left(x, t, \phi, \phi_{t}, \ldots\right)=-\beta \phi_{t}+f(x, t)$ is a kink centered at $X(t)$ whose width $l(t)$ depends on time [Eqs. (5) and (6)], then Eqs. (A3) and (A4) become

$$
\frac{d P}{d t}=-\beta P-\int_{-\infty}^{+\infty} d x f(x, t) \phi_{x}, \quad P(t)=\frac{M_{0} l_{0} \dot{X}(t)}{l(t)}
$$

and

$$
\begin{aligned}
& \frac{P(t) l(t)}{M_{0} l_{0}}\left[\frac{d P}{d t}+\beta P(t)+\int_{-\infty}^{+\infty} d x f(x, t) \phi_{x}\right] \\
& +\dot{i}\left[\frac{P(t)^{2}}{2 M_{0} l_{0}}+\frac{1}{2} \alpha M_{0} l_{0}\left(\frac{2 \ddot{l}}{l}-\frac{\dot{l}^{2}}{l^{2}}\right)+\frac{1}{2} M_{0}\left(\frac{1}{l_{0}}-\frac{l_{0}}{l^{2}}\right)\right. \\
& \left.+\beta \alpha \frac{l_{0}}{l} \dot{l}+\int_{-\infty}^{+\infty} d \theta f(l \theta+X(t), t) \theta \phi_{\theta}\right]=0, \quad(\mathrm{~A} 11
\end{aligned}
$$

respectively. The first bracket on the right hand of Eq. (A11) vanishes because of Eq. (A10), so the solution of Eq. (A11) is $i=0$ or

$$
\begin{aligned}
\alpha\left[\dot{l}^{2}-2 l \ddot{l}-2 \beta l i\right]= & \frac{l^{2}}{l_{0}^{2}}\left[1+\frac{P^{2}}{M_{0}^{2}}\right]-1+\frac{2 l(t)^{2}}{M_{0} l_{0}} \\
& \times \int_{-\infty}^{+\infty} d \theta f[\theta l+X(t), t] \theta \phi_{\theta} .
\end{aligned}
$$

In such a way, Eqs. (A10) and (A12) can be interpreted as the equations which the collective coordinates $X(t)[P(t)]$ and $l(t)$ are satisfied, and they are obtained from $d P / d t$ and 
$d H / d t$, respectively. For example, if $f(x, t)=\epsilon \sin \left(\delta t+\delta_{0}\right)$ in Eqs. (A10) and (A12), we recover Eqs. (18) and (20), obtained by applying the GTWA in Sec. II.

As a final remark, we point out that, since we have not used the explicit form for the potential function $U(\phi)$ in any moment, the equivalence between these two abovementioned methods remains true for any other perturbed nonlinear Klein-Gordon equation of the form of Eqs. (2) and (3), when the Hamiltonian of the system is of the form of Eq. (4).
[1] M. Remoissenet, Waves Called Solitons (Springer, Berlin, 1996).

[2] A.C. Scott, Nonlinear Science (Oxford University Press, Oxford, 1999).

[3] Yu.S. Kivshar and B.A. Malomed, Rev. Mod. Phys. 61, 763 (1989).

[4] A. Sánchez and A.R. Bishop, SIAM Rev. 40, 579 (1998).

[5] M.B. Fogel, S.E. Trullinger, A.R. Bishop, and J.A. Krumhansl, Phys. Rev. Lett. 46, 1411 (1977); Phys. Rev. B 15, 1578 (1977).

[6] D.W. McLaughlin and A.C. Scott, Phys. Rev. A 18, 1652 (1978).

[7] The dynamics of the center of the waves in some cases is more complicated than Newtonian; see, e.g., J.-C. Fernandez, J.M. Gambaudo, S. Gauthier, and G. Reinisch, Phys. Rev. Lett. 46, 753 (1981), and Ref. [17].

[8] M.J. Rice, Phys. Rev. B 28, 3587 (1983).

[9] J.C. Fernandez, M.J. Goupil, O. Legrand, and G. Reinisch, Phys. Rev. B 34, 6207 (1986).

[10] E. Majerníková and G. Drobný, Phys. Rev. E 47, 3677 (1993).

[11] S. Jeyadev and J.R. Schrieffer, Synth. Met. 9, 451 (1984).

[12] D.K. Campbell, J.F. Schonfeld, and C.A. Wingate, Physica D 9, 1 (1983).

[13] Yu.S. Kivshar, F. Zhang, and L. Vázquez, Phys. Rev. Lett. 67, 1177 (1991); Phys. Rev. A 46, 5214 (1992).

[14] Z. Fei, V.V. Konotop, M. Peyrard, and L. Vázquez, Phys. Rev. E 48, 548 (1993).

[15] N.R. Quintero, A. Sánchez, and F.G. Mertens, Phys. Rev. Lett. 84, 871 (2000).

[16] For the sG system there have been reports [R. Boesch and C.R. Willis, Phys. Rev. B 42, 2290 (1990)] of an internal quasimode in the phonon band tentatively identified with the Rice mode, but the existence of such a mode has not been confirmed, and therefore the question remains unsolved.

[17] F.G. Mertens, H.J. Schnitzer, and A.R. Bishop, Phys. Rev. B 56, 2510 (1997).

[18] N.R. Quintero and A. Sánchez, Eur. Phys. J. B 6, 133 (1998).

[19] E. Majerníková, Yu.B. Gaididei, and O.M. Braun, Phys. Rev. E 52, 1241 (1995).

[20] J.L. Reid and J.R. Ray, Z. Angew. Math. Mech. 64, 365 (1984).

[21] E. Pinney, Proc. Am. Math. Soc. 1, 681 (1950).

[22] N.W. McLachlan, Theory and Application of Mathieu Functions (Dover, New York, 1964).

[23] S. Wolfram, The MATHEMATICA Book (Cambridge University Press, Cambridge, 1996).

[24] E.T. Whittaker and G.N. Watson, A Course of Modern Analysis (Cambridge University Press, Cambridge, 1984).

[25] A.H. Nayfeh and D.T. Mook, Nonlinear Oscillations (Wiley, New York, 1979).

[26] A. Erdélyi, W. Magnus, F.Oberhettinger, and F.G. Tricomi, Higher Transcendental Functions (McGraw-Hill, New York, 1955), Vol. III.

[27] W.A. Strauss and L. Vázquez, J. Comput. Phys. 28, 271 (1978).

[28] S. Jiménez and L. Vázquez, Appl. Math. Comput. 35, 61 (1990).

[29] H. Segur, J. Math. Phys. 24, 1439 (1983).

[30] N.R. Quintero, A. Sánchez, and F.G. Mertens, Eur. Phys. J. B 16, 361 (2000).

[31] Yu.S. Kivshar, D.E. Pelinovsky, T. Cretegny, and M. Peyrard, Phys. Rev. Lett. 80, 5032 (1998).

[32] N.R. Quintero, A. Sánchez, and F.G. Mertens, Phys. Rev. E 62, R1 (2000). 